Preface

\title{
Editorial Compilation-II
}

\author{
Emmanuel J. Favaloro, PhD, FFSC (RCPA) ${ }^{1}$ Giuseppe Lippi, MD ${ }^{2}$ \\ 1 Department of Haematology, Institute of Clinical Pathology and \\ Medical Research (ICPMR), Westmead Hospital, Westmead, Australia \\ ${ }^{2}$ Section of Clinical Biochemistry, University of Verona, Verona, Italy \\ Semin Thromb Hemost 2016;42:599-602.
}

Welcome to another issue of Seminars in Thrombosis and Hemostasis. This is the second issue published under the "banner" of "editorial compilation." Although Seminars in Thrombosis and Hemostasis is primarily a theme-driven publication, the occasional opportunity arises to publish intermittent issues containing more wide-ranging chapters of current interest and controversy that do not quite match a current themed issue in progress. In addition, we also require a vehicle for publishing the contributions from our Eberhard $\mathrm{F}$. Mammen Young Investigator Award winners. ${ }^{1-5}$ In previous years, we used the "editorial banner" of "hot topics" to share such interesting potpourri of material with our readership. ${ }^{6-12}$ That series has served Seminars in Thrombosis and Hemostasis well, and has been very popular with the readership. ${ }^{1-3,13-16}$ The new series of "editorial compilation" hopes to continue this tradition, ${ }^{17}$ as well as to potentially expand the content more broadly to include the best of unsolicited content as submitted to the journal and after subsequent expert peerreview. It is interesting that the majority of articles in the current issue relate broadly to the "thrombosis" arena.

This issue begins with the contributions from three of the recent past (2015) Eberhard F Mammen Young Investigator Award winners. The first, from Biss, ${ }^{18}$ asks the question "Is venous thromboembolism (VTE) in children preventable?" The incidence of VTE in children is noticeably increasing. Hospitalized infants and adolescents are at highest risk, and most individuals who have VTE also have multiple thrombotic risk factors. The presence of a central venous catheter (CVC) is the most frequent risk factor for childhood thrombosis. Childhood VTE has significant consequences in relation to the thrombotic event and the anticoagulant therapy used for its treatment. Identification of the most prevalent risk factors for VTE, particularly amongst adolescents, has moved the focus toward prevention of thrombosis. Risk assessment models have been developed to identify individuals who are at higher risk with a view to employing preventative strategies such as mechanical and chemical thromboprophylaxis.

Address for correspondence Emmanuel J. Favaloro, PhD, FFSc (RCPA), Department of Haematology, Institute of Clinical Pathology and Medical Research (ICPMR), Westmead Hospital, Westmead, NSW 2145, Australia (e-mail: emmanuel.

favaloro@health.nsw.gov.au).
There is currently little evidence to support the efficacy of such strategies for preventing either CVC-associated thrombosis or thrombosis at other sites. In addition, there are concerns about adverse consequences of mechanical and chemical thromboprophylaxis in a population where the overall incidence of VTE remains globally low.

Moving from children to potential mothers, the second contribution, from Stanciakova et $\mathrm{al}^{19}$ is on the monitoring of hemostasis and management of anticoagulant thromboprophylaxis in pregnant women with increased risk of fetal loss. Physiological prothrombotic changes during pregnancy and the postpartum period, along with other preexisting maternal risk factors, increase the risk of both VTE and adverse pregnancy outcomes. Pregnancy complications developing due to placental insufficiency as a result of inappropriate activation of coagulation are present in more than $5 \%$ of pregnancies and can contribute to significant maternal morbidity and mortality. Therefore, anticoagulant prophylaxis in women with congenital and acquired thrombophilic conditions should be actively considered. According to the Guidelines of American College of Chest Physicians, the use of lowmolecular-weight heparin is suggested for prophylaxis of VTE and pregnancy complications in high-risk pregnant women. However, personalized refinements of such thromboprophylaxis remains unspecified, despite the necessity of better targeted recommendations for life-threatening conditions. The authors therefore review the possibilities of longitudinal monitoring and comprehensive assessment of changes in hemostasis in the group of high-risk pregnant women, which can then be used for early prediction and individualization of the optimal anticoagulant thromboprophylaxis of pregnancy complications. They also present their single-center experience with such monitoring.

The third contribution is also from a 2015 Young Investigator Award winner, Coutinho, and a team of his coworkers, and is on the topic of risk factors for cerebral venous thrombosis (CVT), ${ }^{20}$ a rare thrombotic disorder involving the
Issue Theme Editorial

Compilation II; Guest Editors: Emmanuel J. Favaloro, PhD, FFSc (RCPA) and Giuseppe Lippi, MD.
Copyright (c) 2016 by Thieme Medical Publishers, Inc., 333 Seventh Avenue, New York, NY 10001, USA. Tel: +1(212) 584-4662.
DOI http://dx.doi.org/ 10.1055/s-0036-1588011. ISSN 0094-6176. 
cerebral veins and dural sinuses. In contrast to more common sites of VTE, such as the legs and lungs, CVT mainly affects young adults and children, and women are affected three times more often than men. Although presenting symptoms are variable, headache is usually the first, often in combination with focal neurological deficits and epileptic seizures. The primary therapy for CVT consists of heparin followed by oral anticoagulation for at least 3 to 6 months. The mortality in the acute phase is 5 to $10 \%$ and a substantial proportion of survivors suffer from long-term disabilities. A large number of risk factors have been linked to CVT, although the scientific evidence for an association varies considerably between risk factors. Some of these, such as hereditary thrombophilia, correspond with risk factors for most common sites of VTE, whereas others, such as head trauma, are specific for CVT. In most patients, at least one risk factor can be identified.

The next series of chapters comprise either contributions from editorial members of Seminars in Thrombosis and Hemostasis, or the best of recent unsolicited material, but essentially all relate to the "prothrombotic" theme. Lippi et al start the series by reporting that the mean platelet volume (MPV) is decreased in patients diagnosed with VTE in the emergency department. ${ }^{21}$ Platelets play an essential role in hemostasis and thrombosis, and hyperactive platelets are involved in the pathogenesis of cardiovascular disorders. Nevertheless, the role of platelet size, as a biological marker of platelet activation, remains debated in the setting of VTE. A retrospective case-control study was performed to clarify the potential association between an MPV and newly diagnosed VTE by reviewing data of all consecutive patients receiving this diagnosis at the emergency department of a large academic hospital during an entire year. Outpatients undergoing routine laboratory testing for health check-up at the same hospital during the same period represented the control population. The MPV was comparatively decreased in the entire cohort of patients with VTE compared with the outpatient population, as well as in those with isolated deep vein thrombosis (DVT) or pulmonary embolism (PE). A decreased MPV value was found to be associated with an increased risk of VTE, as well as of isolated DVT and isolated PE. A decreased MPV value in active cancer patients was associated with the highest risk of thrombosis. These results describe an inverse association between an MPV and the risk of VTE at diagnosis, thus corroborating the results of other studies published on this topic.

Mehdipoor et al then present a systematic review on PE as a consequence of ultrasonographic examination of extremities for suspected VTE. ${ }^{22}$ Although ultrasonographic examination for DVT appears to be a safe diagnostic method, there remains a theoretical concern that dislodgment of thrombi during examination may occur and lead to PE. The authors therefore conducted a systematic review of the literature to identify reports of possible or confirmed PE as a consequence of ultrasonographic assessment of extremities in patients with suspected DVT. They searched PubMed for studies published in English from January 1960 to April 2015, and included all cohort studies, case series, and case reports that described PE as a consequence of ultrasonographic ssessment of extremities, but excluded those that reported assessment of areas other than the extremities. The authors screened 3,626 articles, 15 of which reported the issue of clot dislodgement and embolization following ultrasonographic examination of the extremities, including 8 original case reports ( 7 men and 1 woman). DVTs were in the lower extremities in all eight cases; five in right and three in left lower extremity. In six cases the femoral veins were involved, and a free-floating thrombus was reported in two cases. Compression ultrasonography was used in all cases, with or without adjunct techniques. Overall, there were seven confirmed and one probable PE cases, two of which had fatal outcomes. The authors conclude that clot embolization is a rare but potential complication of ultrasonic examination for DVT that can lead into PE, and that radiologists and clinicians should be aware of this potentially serious phenomenon and avoid excessive pressure when performing ultrasonographic studies of the extremities.

Next, Spirk et $\mathrm{al}^{23}$ report on data from the Swiss Venous Thrombo Embolism Registry (SWIVTER) on the association between cancer and VTE in regards to adverse clinical outcomes requires. They compared outcomes between 493 patients with cancer-associated VTE and 1,569 VTE patients without cancer, and identified independent predictors of 90-day mortality. Among cancer patients, 351 (71\%) had active disease at the time of VTE diagnosis, and 232 (47\%) had metastatic disease. Cancer patients more frequently had asymptomatic VTE, iliofemoral and upper extremity DVT. Cancer was associated with an increased risk of cumulative 90-day mortality, recurrent VTE, and bleeding requiring medical attention. Among cancer patients, the strongest factor associated with mortality was metastatic disease, whereas PE represented the strongest factor among noncancer patients. Symptomatic as compared with asymptomatic VTE predicted neither mortality nor recurrent VTE in cancer patients. The authors conclude that in regards to these data, early mortality of cancer-associated VTE was mainly driven by the extent of cancer disease and not by VTE symptoms or severity.

Silvestri et al then pose another question of whether there is a role for immunosuppressive treatments in thrombosis associated with autoimmune diseases. ${ }^{24}$ Autoimmune diseases are not infrequently associated with arterial or venous thrombotic events, and chronic inflammation and immune system impairment are considered the main pathogenetic mechanisms. Some of the drugs used in the treatment of such diseases have been associated with an increased risk of thrombosis. On the other hand, their anti-inflammatory and immune modulator activity could correct some of the mechanisms otherwise leading to thrombosis. In this review, the authors examine recent available evidence and report a lack of adequate studies. Nevertheless, the available evidence suggests that glucocorticoids and high-dose immunoglobulins are associated with an increased incidence of VTE. The authors conclude that although available data do not permit definite conclusions and more data are needed from future studies and registries, physicians should be aware of these associations. 
Elewa et al then report on triple oral antithrombotic therapy (TOAT) in atrial fibrillation and coronary artery stenting, and the search for the best combination of treatment. ${ }^{25}$ Patients with atrial fibrillation (AF) who are treated with oral anticoagulants often have concurrent coronary artery disease. TOAT has been often necessary to prevent stent thrombosis or myocardial infarction associated with percutaneous coronary intervention or acute coronary syndrome in patients with comorbid coronary artery disease and AF. While the use of TOAT (aspirin, clopidogrel, and a vitamin $\mathrm{K}$ antagonist [VKA] such as warfarin) has excellent efficacy against thrombotic complications, this comes at the expense of increased bleeding risk. This review discusses potential strategies to improve TOAT benefit-risk ratio according to evidence from the literature. These strategies include: (1) dropping aspirin; (2) reducing the duration of TOAT; (3) switching warfarin to a direct oral anticoagulant (DOAC); (4) the use of DOAC in combination with a single antiplatelet agent; and (5) switching clopidogrel to a novel antiplatelet agent. The authors conclude that while dropping aspirin and reducing TOAT duration should be considered in selected AF patients at low risk of thrombosis, the role of DOACs and novel antiplatelet agents in TOAT has not been thoroughly studied and there is limited evidence to support their use currently. They further suggest that ongoing studies will provide safety and efficacy data to guide clinicians who frequently face the challenge of determining the best TOAT combination for their patients.

Continuing the theme of anticoagulant therapy, Dentali et al review the effect of vitamin $\mathrm{K}$ intake on the stability of treatment with VKAs. ${ }^{26}$ VKAs are highly effective for the primary and secondary prevention of arterial and venous thromboembolic events. However, patients treated with VKA have on average only $60 \%$ of their international normalized ratio (INR) values within the therapeutic range and INR instability is associated with an increased risk of thrombosis and bleeding events. Recent evidence suggests that poor dietary vitamin $\mathrm{K}$ intake may affect anticoagulation control, but the role of vitamin $\mathrm{K}$ in INR stability remains to be established. The authors performed a systematic review of the literature to assess the role of vitamin $\mathrm{K}$, dietary intake on the stability of VKA, and the potential effect of daily vitamin $\mathrm{K}$ supplementation on VKA therapy. After a search in Medline and Embase databases, 15 studies for a total of 1,838 patients were included in their systematic review. Observational studies suggest an increased risk of unstable anticoagulation control in patients with lower daily vitamin $\mathrm{K}$ intake. On the other hand, the role of daily vitamin $\mathrm{K}$ supplementation or a diet with controlled vitamin $\mathrm{K}$ content in patients on VKA treatment remains to be established. Use of daily vitamin $\mathrm{K}$ supplementation may be associated with a clinically relevant increase in the time in therapeutic range in patients with unstable anticoagulation control. Conversely, this effect appears small and not clinically relevant when vitamin $\mathrm{K}$ was administered to an unselected population receiving VKA. The authors conclude that other large prospective studies are necessary to confirm these preliminary findings.
Finally, Danese et al ask the last question for this issue of Seminars in Thrombosis and Haemostasis, and specifically is coagulation factor XII (FXII) an active player or "innocent" bystander in hemostasis and thrombosis? ${ }^{27}$ Formerly known as Hageman factor, FXII is a plasma glycoprotein that exerts a kaleidoscope of biological functions, including the initiation of the intrinsic pathway of blood coagulation, the activation of the kallikrein-kinin system and the generation of bradykinin and angiotensin. The large body of evidence accumulated over the past decades and the revised cell-based model of hemostasis suggests that FXII might somehow be considered "redundant" for physiological hemostasis, thereby drawing a potential interpretation that this protein is an 'innocent' bystander of in vivo hemostasis. Moreover, although the contribution of FXII remains unproven in the pathogenesis of VTE, perhaps reinforcing this perception of "redundancy," more recent work identifies FXII as critical for initiation of thrombosis on artificial surfaces (e.g., polyurethanes or polytetrafluoroethylene catheters), or in patients with strong prothrombotic conditions such as vulnerable atherosclerotic plaques or severe bacterial infections. Important evidence has also emerged from recent investigations using innovative FXII inhibitors in ex vivo animal models, wherein targeted FXIImediated inhibition of thrombin and fibrin generation may open new avenues for prevention or treatment of certain types of thrombosis. This conclusion is supported by similar evidence published for targeted inhibition of factor XI (FXI). ${ }^{28}$ Thus, interest in FXII, waned in the recent past, appears to again be reemerging, and pointing to an important but underrecognized contribution of FXII to in vivo hemostasis and thrombosis.

As always, we would like to thank all the authors to this new issue of "editorial compilations" for their original and comprehensive contributions. We also hope that you, representing the readership of this journal, find this issue of substantial interest. This will of course be determined in time, as measured and established in previous issues of this journal. $^{1-3,13-16}$

\section{References}

1 Favaloro EJ. 2011 Eberhard F. Mammen award announcements. Semin Thromb Hemost 2011;37(5):431-439

2 Favaloro EJ. 2012 Eberhard F. Mammen award announcements. Semin Thromb Hemost 2012;38(5):425-432

3 Favaloro EJ. 2013 Eberhard F. Mammen award announcements. Semin Thromb Hemost 2013;39(6):567-574

4 Favaloro EJ. 2014 Eberhard F. Mammen award announcements: part II-Young Investigator Awards. Semin Thromb Hemost 2014; 40(7):718-723

5 Favaloro EJ. 2015 Eberhard F. Mammen award announcements: part II-young investigator awards. Semin Thromb Hemost 2015; 41(8):809-815

6 Favaloro EJ. Hot topics I: A potpourri of current issues and controversies in thrombosis and hemostasis. Semin Thromb Hemost 2007;33(8):723-726

7 Favaloro EJ. Hot topics II: an editorial collection of current issues and controversies in thrombosis and hemostasis. Semin Thromb Hemost 2008;34(1):3-6 
8 Favaloro EJ. Hot topics III. Preface. Semin Thromb Hemost 2012; 38(1):1-4

9 Favaloro EJ. Hot Topics IV. Semin Thromb Hemost 2013;39(1):1-4

10 Favaloro EJ. Hot Topics V. Semin Thromb Hemost 2014;40(1):5-10

11 Favaloro EJ. Hot topics VI. Preface. Semin Thromb Hemost 2014; 40(7):713-717

12 Favaloro EJ. Hot Topics VII. Semin Thromb Hemost 2015;41(4): 355-358

13 Favaloro EJ. Editorial. Winners of the inaugural Eberhard F. Mammen award for most popular article. Semin Thromb Hemost 2009; 35(7):587-590

14 Favaloro EJ. Winners of the 2010 Eberhard F. Mammen award for most popular article during 2008-2009. Semin Thromb Hemost 2010;36(7):685-692

15 Favaloro EJ. 2014 Eberhard F. Mammen award announcements: Part I -most popular articles. Semin Thromb Hemost 2014;40(4):407-412

16 Favaloro EJ. 2015 Eberhard F. Mammen award announcements: Part I -most popular articles. Semin Thromb Hemost 2015;41(7):673-679

17 Favaloro EJ, Lippi G. Editorial compilation I. Semin Thromb Hemost 2016;42(1):5-8

18 Biss TT. Venous thromboembolism in children: Is it preventable? Semin Thromb Hemost 2016;42(6):603-611

19 Stanciakova L, Dobrotova M, Jedinakova Z, et al. Monitoring of hemostasis and management of anticoagulant thromboprophylaxis in pregnant women with increased risk of fetal loss. Semin Thromb Hemost 2016;42(6):612-621

20 Silvis SM, Middeldorp S, Zuurbier SM, Cannegieter SC, Coutinho JM. Risk factors for cerebral venous thrombosis. Semin Thromb Hemost 2016;42(6):622-631
21 Lippi L, Buonocore R, Cervellin G. The mean platelet volume is decreased in patients diagnosed with venous thromboembolism in the emergency department. Semin Thromb Hemost 2016; 42(6):632-635

22 Mehdipoor G, Shabestari AA, Lip GYH, Bikdeli B. Pulmonary embolism as a consequence of ultrasonographic examination of extremities for suspected venous thrombosis: a systematic review. Semin Thromb Hemost 2016;42(6):636-641

23 Spirk D, Aujesky D, Stuck AK, et al. Clinical outcomes of venous thromboembolism in patients with and without cancer: The SWIss Venous ThromboEmbolism Registry (SWIVTER). Semin Thromb Hemost 2016;42(6):642-649

24 Silvestri E, Scalera A, Emmi G, et al. Thrombosis in autoimmune diseases: a role for immunosuppressive treatments? Semin Thromb Hemost 2016;42(6):650-661

25 Elewa H, Ahmed D, Barnes GD. Triple oral antithrombotic therapy in atrial fibrillation and coronary artery stenting: Searching for the best combination. Semin Thromb Hemost 2016;42(6):662-670

26 Dentali F, Crowther M, Galli M, et al; for the WARPED investigators. Effect of vitamin $\mathrm{K}$ intake on the stability of treatment with vitamin K antagonists: a systematic review of the literature. Semin Thromb Hemost 2016;42(6):671-681

27 Danese E, Montagnana M, Lippi G. Factor XII in hemostasis and thrombosis. Active player or (innocent) bystander? Semin Thromb Hemost 2016;42(6):682-688

28 Lippi G, Harenberg J, Mattiuzzi C, Favaloro EJ. Next generation antithrombotic therapy: focus on antisense therapy against coagulation factor XI. Semin Thromb Hemost 2015;41(2): 255-262 